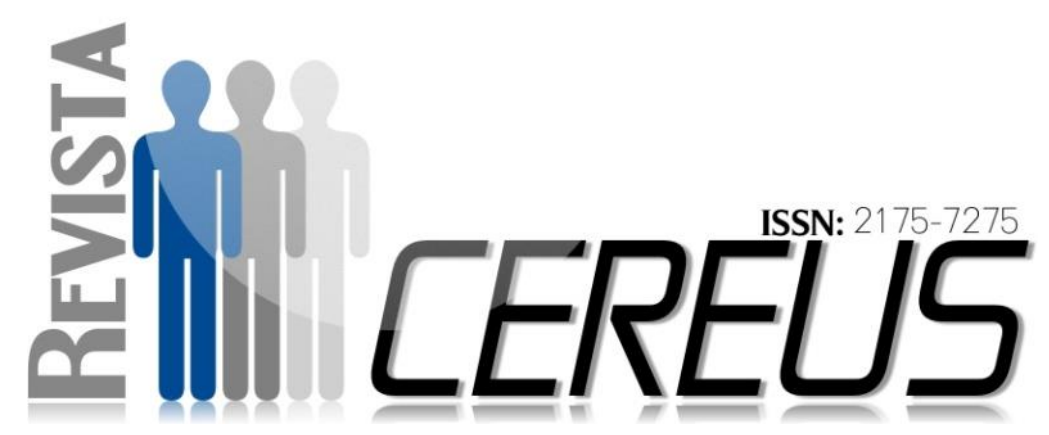

DOI: 10.18605/2175-7275/cereus.v9nep2-18.

\title{
SOFTWARES PARA SUPORTE NO ENSINO DE ENGENHARIA CIVIL: UM MAPEAMENTO SISTEMÁTICO DOS SEUS USOS NAS INSTITUIÇÕES BRASILEIRAS
}

\author{
PASSOS, Denis da Silva ${ }^{1}$; \\ VENEGA, Virginia de Sousa ${ }^{\prime}$; \\ ROCHA, Marcelo Lisboa ${ }^{2}$.
}

\section{RESUMO}

Nas últimas décadas, a Engenharia Civil muito tem evoluído, especialmente com relação ao uso da informática. No âmbito educacional é possível constatar um expressivo número de softwares para o ensino nesta área. Nessa perspectiva objetivamos realizar um Mapeamento Sistemático de Literatura (MSL) sobre o uso de softwares para auxílio educacional em cursos de Engenharia Civil no Brasil. Com a intenção de ser uma revisão formal, rigorosa e confiável, a execução do MSL teve por base a metodologia de Revisões Sistemáticas, a qual se mostrou adequada para a obtenção de estudos relevantes e geração de dados necessários para responder às questões de pesquisa estabelecidas. Foram utilizadas as bases de dados do Congresso Brasileiro de 1 Alunos do Programa de mestrado em modelagem Computacional de sistemas
pela Universidade Federal do Tocantins
2 Doutorado. Engenharia Elétrica Universidade Federal do Rio de Janeiro,
UFRJ, Professor do Programa de mestrado em modelagem Computacional de
Sistemas pela Universidade Federal do Tocantins. E- mail:
marcelolisboarocha@gmail.com 
Educação em Engenharia, Revista Brasileira de Informática na Educação, Revista de Ensino de Engenharia e Simpósio Brasileiro de Informática na Educação. Foram encontrados 69 trabalhos científicos, dos quais 62 foram incluídos no MSL. Nestes estudos foram identificados 55 diferentes softwares sendo que os mais recorrentes foram Matlab, Excel, Scilab, Ftool, GDFácil, Geogebra, GEOWEB, Mathematica e Winplot. As principais áreas de aplicação dos softwares foram: Cálculo; Estruturas; Estruturas Metálicas; Geotecnia; Mecânica dos Solos; Análise de Estruturas e Desenho Técnico. Dentre as ferramentas identificadas houve predominância daquelas para desktop (87\%) com interface GUI 2D (50\%).

Palavras chave: Educação. Engenharia Civil. Ensino. Interdisciplinar. Mapeamento Sistemático. Softwares.

\section{SOFTWARES FOR SUPPORT IN CIVIL ENGINEERING EDUCATION: A SYSTEMATIC MAPPING OF ITS USES IN BRAZILIAN INSTITUTIONS}

\section{ABSTRACT}

In recent decades, Civil Engineering has evolved a lot, especially in relation to the use of information technology. In the educational field it is possible to observe an expressive number of software for teaching in this area. From this perspective we aim to perform a Systematic Literature Mapping (SLM) on the use of softwares for educational aid in Civil Engineering courses in Brazil. With the intention of being a formal, rigorous and reliable review, the implementation of the MSL was based on the methodology of Systematic Reviews, which proved adequate to obtain relevant studies and generation of data necessary to answer the established research questions. We used the databases of the Brazilian Congress of Engineering Education, Brazilian Journal of Informatics in Education, Engineering Teaching Journal and 
Brazilian Symposium on Informatics in Education. A total of 69 scientific papers were found, of which 62 were included in the SLM. The total number of authors was 154. In these studies, 55 different software were identified and the most recurrent were Matlab, Excel, Scilab, Ftool, GDFácil, Geogebra, GEOWEB, Mathematica and Winplot. The main application areas of the software were: Calculation; Structures; Metallic structures; Geotechnics; Soil Mechanics; Analysis of Structures and Technical Design. Among the identified tools there were predominance of those for desktop (87\%) with GUI 2D interface (50\%).

Keywords: Civil Engineering. Teaching. Systematic Mapping. Softwares 


\section{INTRODUÇÃO}

Nas últimas décadas o processo de ensino transformou-se sobremaneira, em grande parte devido à popularização do computador e das ferramentas de Tecnologia da Informação e Comunicação (TIC). Nessa nova realidade, o aluno é capaz de utilizar seu conhecimento prévio aliado às novas tecnologias disponíveis para facilitar a construção do conhecimento (Dominicini e Coelho, 2014).

Estas mudanças, que não se referem somente à tecnologia ou a educação, são tratadas por Castells (1998), que discorre sobre os efeitos fundamentais das TIC no mundo contemporâneo, os quais se estendem à economia, cultura, trabalho, concepção de tempo e espaço, a globalização, Estado e política.

Para LAGOS (2000), um dos desafios atuais da educação é aproveitar as novas mídias (áudio, computadores, inteligência artificial, realidade virtual, vídeo, etc) para atrair o estudante, permitindo a construção do conhecimento.

A Engenharia Civil também tem passado por um período de grande desenvolvimento tecnológico, especialmente com relação ao uso da informática (Modler e Feil, 2003). Evidentemente essa evolução também se faz necessária na educação. $E$ já é possível constatar um grande número de aplicações computacionais voltadas para o ensino de Engenharia Civil em suas mais diversas vertentes.

Num aspecto amplo, Valente (1999) destaca que quando o aluno usa - computador para construir o seu conhecimento, o computador se torna uma ferramenta de ensino oferecendo condições para o aluno apresentar a resolução de problemas, refletir sobre resultados e depurar suas ideias.

Por sua vez, Ormonde (2013) antecedido por Silva et al. (2005) apresentam diversos softwares educacionais voltados para áreas da Engenharia Civil e o segundo autor aponta que:

“...o emprego dessas ferramentas gráficas faz com que os alunos de graduação sintam-se mais motivados para estudar os assuntos discutidos em sala de aula, o que torna 0 aprendizado mais dinâmico, interativo e eficiente, visto que estes passam a visualizar e compreender com mais clareza o comportamento de sistemas estruturais de engenharia, como também os fenômenos físicos envolvidos." (SILVA et al., 2005, p.53). 
Ramiro, Costa e Bernardes (2014) pontuam que nas disciplinas essenciais para a formação dos alunos de Engenharia Civil, (por exemplo: Mecânica dos Sólidos, Isostática e Hiperestática), a Informática pode ser utilizada como ferramenta motivadora, auxiliando no desenvolvimento cognitivo, e trabalhando conceitos e fundamentos aprendidos em outras disciplinas. Ou seja, a utilização adequada de ferramentas computacionais cria um cenário propício à interdisciplinaridade, pois há "necessidade de transferência de métodos de uma disciplina para outra" (NICOLESCU, 1999) bem como "intercâmbio de conceitos,

\section{METODOLOGIA}

Com o objetivo de identificar, catalogar e avaliar os estudos científicos que tratam sobre o uso de softwares para auxílio educacional em cursos de Engenharia Civil no Brasil, foi realizado um Mapeamento Sistemático de conhecimentos e métodos entre as disciplinas" (JANTSCH; BIANCHETTI, 1999).

Neste contexto, o objetivo deste trabalho foi realizar um Mapeamento Sistemático de Literatura (MSL) sobre o uso de softwares para auxílio educacional em cursos de Engenharia Civil no Brasil.

Um MSL é um tipo de Revisão Sistemática, onde se realiza uma revisão ampla dos estudos, buscando identificar as evidências disponíveis, bem como identificar lacunas e áreas para direcionar futuras revisões sistemáticas ou mais estudos primários (KITCHENHAM, 2004; PETERSEN, 2008).

Literatura (MSL). A realização deste MSL foi baseada na metodologia exposta por Kitchenham e Charters(2007) e está dividida em três fases: Fase 1 - Planejamento; Fase 2 Condução; Fase 3 - Documentação. 


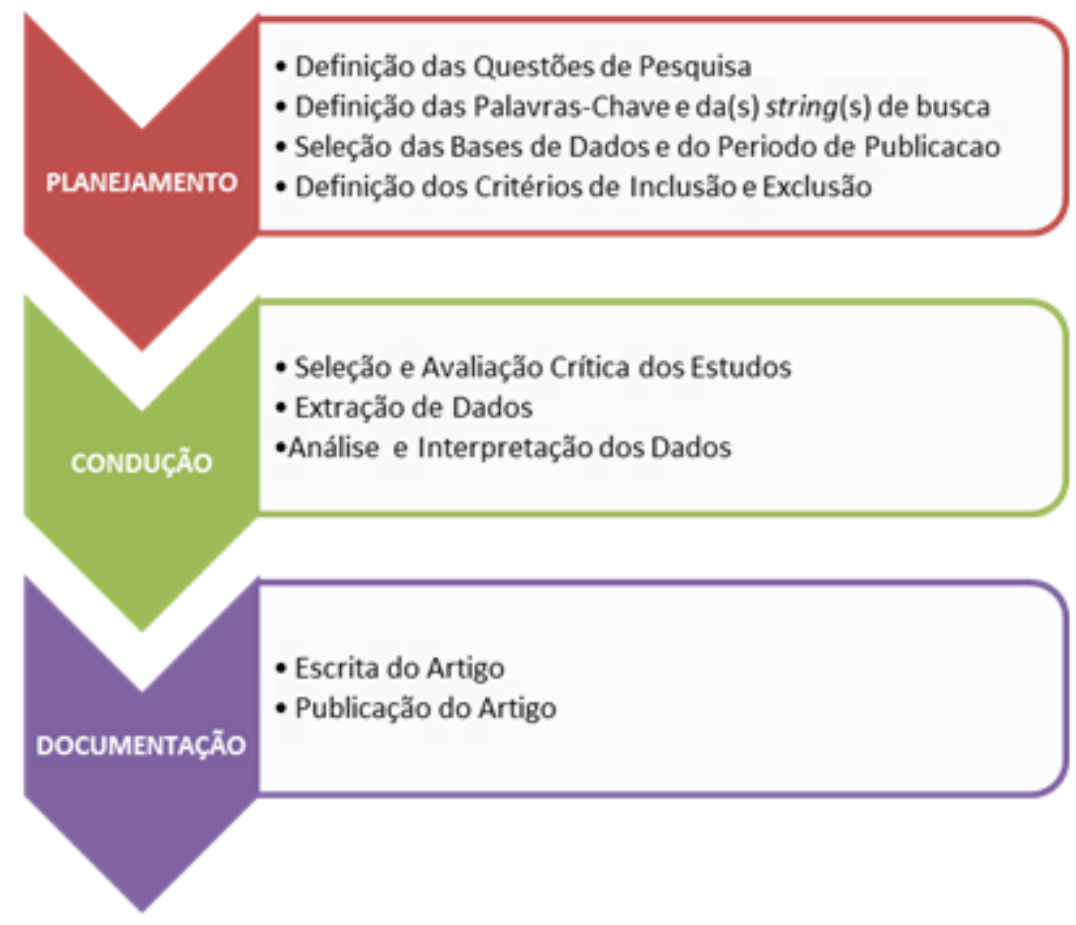

Figura 1: Visão geral da metodologia adotada neste Mapeamento Sistemático

\subsection{PLANEJAMENTO DO MAPEAMENTO SISTEMÁTICO}

Esta seção expõe a Fase 1 (Planejamento) do MSL realizado neste trabalho, de acordo com o modelo proposto por Kitchenham e Charters (2007).

No intuito de nortear a realização do presente trabalho, foram definidas 4

\section{Tabela 1: Questões de Pesquisa para o Mapeamento Sistemático}

(quatro) questões de pesquisa. Tais perguntas estão contidas na Tabela 1 acompanhadas de um código por meio do qual serão referências no decorrer deste artigo.

\begin{tabular}{ll}
\hline REF & \multicolumn{1}{c}{ QUESTÃO DE PESQUISA } \\
\hline QP1 & Quais softwares têm sido utilizados no ensino de Engenharia Civil no Brasil? \\
QP2 & $\begin{array}{l}\text { Em quais temas/disciplinas dos cursos de Engenharia Civil o uso dos softwares é } \\
\text { mais frequente? }\end{array}$ \\
QP3 & $\begin{array}{l}\text { Quais tipos de interfaces ou tecnologias de interação usuário-computador são } \\
\text { usadas nos softwares identificados na QP1? }\end{array}$ \\
QP4 & $\begin{array}{l}\text { Quais as principais plataformas para as quais os softwares identificados na QP1 } \\
\text { têm sido desenvolvidos? }\end{array}$ \\
\hline
\end{tabular}


A partir da questão QP1, as palavraschave deste MSL foram definidas e são destacadas a seguir:

Quais softwares têm sido utilizados no

\subsubsection{Definição das Strings de Busca}

Com base nas palavras-chave anteriormente expostas, a string de busca pôde então ser definida. Para tal, conforme Tabela 2, foram utilizados operadores lógicos OR e AND para ensino de Engenharia Civil no Brasil?

Ou seja:

"software", "ensino", "engenharia civil

Tabela 2: Palavras-chave e respectivas substrings correspondentes

\begin{tabular}{cl}
\hline PALAVRA-CHAVE & \multicolumn{1}{c}{ SUBSTRING RELACIONADA } \\
\hline software & software OR ferramenta OR aplicativo OR jogo OR game OR "aplicação \\
& computacional" OR computador OR virtual OR sistema \\
ensino & ensino OR aprendizagem OR educação OR educacional \\
engenharia civil & engenharia civil \\
\hline
\end{tabular}

String resultante:

(software OR ferramenta OR aplicativo OR jogo OR game OR “aplicação computacional” OR

\subsubsection{Seleção das Bases de Dados}

Com o propósito de encontrar os trabalhos mais relacionados com 0 tema deste MSL, foram realizadas combinar os termos das palavraschave com alguns sinônimos e expressões relacionadas com o tema, bem como variações (plural e singular). 
Tabela 3: Bases de dados selecionadas para o MSL

\begin{tabular}{ll}
\hline \multicolumn{1}{c}{ BASE DE DADOS } & \multicolumn{1}{c}{ LINKS } \\
\hline $\begin{array}{l}\text { Congresso Brasileiro de Educação em } \\
\text { Engenharia - COBENGE }\end{array}$ & abenge.org.br/cobenges-anteriores/ \\
$\begin{array}{l}\text { Revista Brasileira de Informática na } \\
\text { Educação - RBIE }\end{array}$ & www.br-ie.org/pub/index.php/rbie \\
Revista de Ensino de Engenharia - REE & www.abenge.org.br/revista/index.php/abenge/index \\
$\begin{array}{l}\text { Simpósio Brasileiro de Informática na } \\
\text { Educação - SBIE }\end{array}$ & www.br-ie.org/pub/index.php/sbie/issue/archive \\
\hline
\end{tabular}

O método utilizado para recuperação dos estudos consistiu em realizar buscas manuais nos anais do

\subsubsection{Período de Publicação}

Não houve delimitação de intervalo de tempo nas buscas deste MSL. As buscas corresponderam à disponibilidade e/ou tempo de

\section{. 2.1.5. Critérios de Inclusão e Exclusão}

Foram adotados os seguintes critérios para inclusão das publicações no arcabouço analisado:

Cl-1: Os trabalhos devem versar sobre uso de softwares para auxílio educacional em cursos de Engenharia Civil no Brasil, apresentando relatos de experiências em ensino-aprendizagem com os softwares, ou sugestões de uso de softwares para áreas da Engenharia Civil, ou propostas de softwares.
COBENGE; e buscas através dos mecanismos próprios nos websites das revistas RBIE e REE e do SBIE.

existência de cada evento ou periódico, abrangendo todas as publicações acessíveis 


\subsection{CONDUÇÃO DO MAPEAMENTO SISTEMÁTICO}

Uma vez definido o Planejamento do MSL, procedeu-se à execução (Condução) propriamente dita. Esta fase foi composta por três etapas: 1 - Seleção e Avaliação Crítica dos Estudos; 2 - Extração dos Dados; 3 - Análise e Interpretação dos Dados.
A seguir são detalhadas as etapas 1 e 2 visto que a terceira se demonstra nos desdobramentos da Seção 3 (Resultados).

\subsubsection{Seleção e Avaliação Crítica dos Estudos}

Os estudos que compuseram o arcabouço analisado neste MSL foram classificados em cinco conjuntos no decorrer de três etapas que atuaram como filtros consecutivos, conforme descrito a seguir:

Etapa 1 - Identificação: Na intenção de identificar os estudos que possuíssem potencial relação com o tema, foram executadas buscas nas bases de dados com a utilização da string definida para o trabalho (vide subseção 2.1.2). Desta etapa resultou o conjunto que aqui denominamos Estudos identificados, do qual se registrou apenas a quantidade de itens.

Etapa 2 - Seleção: Realizou-se então leitura do título, resumo e palavraschave dos estudos identificados na etapa anterior, analisando quais atendiam previamente os critérios estabelecidos na subseção 2.1.5. Como resultado, obtivemos dois conjuntos: Estudos Selecionados e Estudos Não Selecionados (registrada apenas a quantidade de itens).

Etapa 3 - Inclusão: Nesta etapa, foi realizada leitura minuciosa do texto integral dos trabalhos selecionados anteriormente e reaplicados os critérios de inclusão/exclusão. Como consequência, foram compostos os dois últimos conjuntos: Estudos Incluídos (de onde foi realizada a extração dos dados) e Estudos Excluídos.

A Figura 2 ilustra as etapas aqui expostas. 


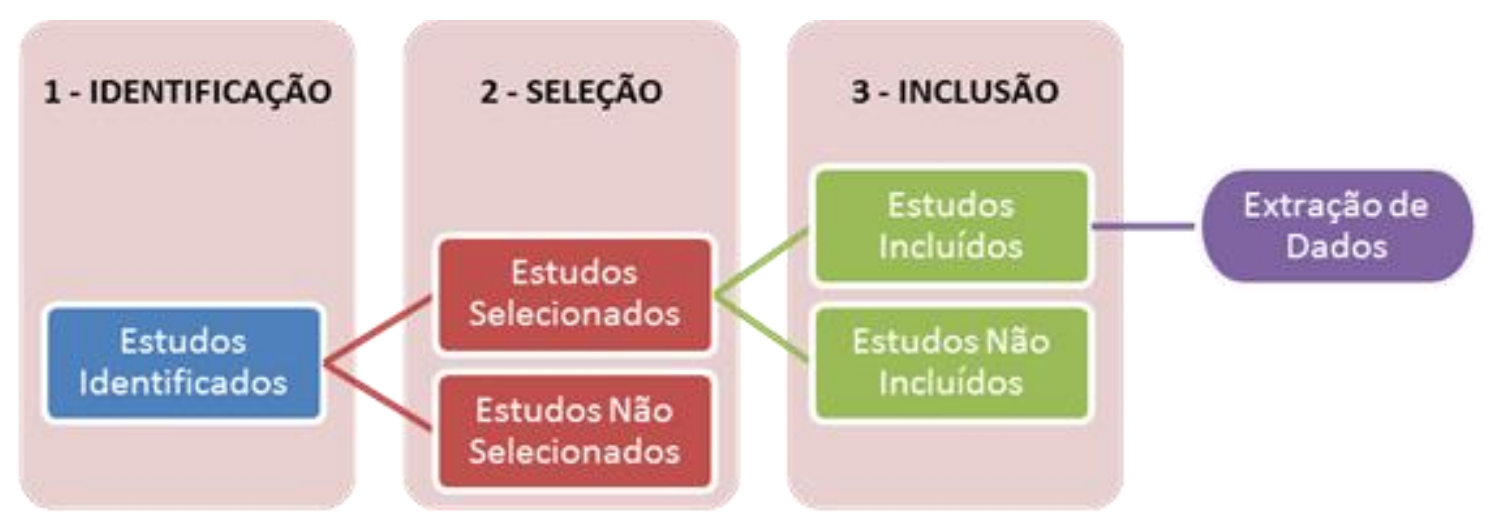

Figura 2: Etapas e conjuntos da seleção e avaliação crítica dos estudos

\subsubsection{Extração dos Dados}

Por fim, procedeu-se à extração dos dados dos Estudos Incluídos. Estes dados foram armazenados em uma planilha possibilitando posterior análise e interpretação.

\section{RESULTADOS}

As buscas realizadas nas bases escolhidas resultaram num conjunto final composto de 62 trabalhos acadêmicos (Estudos Incluídos). Este conjunto foi analisado para obtenção das respostas às questões de pesquisa elencadas na Tabela 1.

Os 62 trabalhos foram escritos por 154 autores de diversas instituições, os quais utilizaram 174 palavras-chave descritoras.

Tabela 4: Resultados gerais

\begin{tabular}{lc}
\hline & QUANTIDADE \\
\hline Autores & 154 \\
Estudos incluídos & 62 \\
Palavras-chave & 174 \\
\hline
\end{tabular}

Para simplificação do texto, no decorrer deste trabalho utilizaremos apenas ID's (exemplo: E15) para cada estudo incluído. A lista com todos os ID's encontra-se disponível em https://goo.gl/KZ6M9T. 
A Tabela 5 apresenta uma visão detalhada da filtragem dos trabalhos a cada etapa da Seleção e Avaliação Crítica dos Estudos.

Tabela 5: Total de estudos por etapa da Seleção e Avaliação Crítica

\begin{tabular}{|c|c|c|c|c|}
\hline \multirow[b]{2}{*}{ BASE } & \multicolumn{3}{|c|}{ SELEÇÃO E AVALIAÇÃO CRÍTICA } & \multirow{2}{*}{ ESTUDOS INCLUÍDOS } \\
\hline & ETAPA 1 & ETAPA 2 & ETAPA 3 & \\
\hline COBENGE & 61 & 57 & 55 & $\begin{array}{c}\text { E1, E2, E3, E4, E5, E6, E7, E8, E9, E10, E12, } \\
\text { E13, E14, E15, E16, E17, E18, E19, E20, E21, } \\
\text { E23, E24, E25, E26, E27, E29, E30, E32, E33, } \\
\text { E34, E35, E36, E37, E39, E41, E42, E43, E44, } \\
\text { E45, E46, E47, E48, E49, E50, E51, E52, E53, } \\
\text { E54, E55, E56, E58, E59, E60, E61, E62 }\end{array}$ \\
\hline RBIE & 0 & 0 & 0 & - \\
\hline REE & 5 & 5 & 5 & E22, E28, E38, E40, E57 \\
\hline SBIE & 3 & 2 & 2 & $\mathrm{E} 11, \mathrm{E} 31$ \\
\hline TOTAL & 69 & 64 & 62 & \\
\hline
\end{tabular}

\section{A Figura 3 apresenta a} distribuição temporal dos 62 trabalhos incluídos. Foi verificado que a quantidade de trabalhos publicados sobre o tema oscilou entre dois e oito por ano, sendo que, nos anos de 2001, 2002, 2011 e 2015 nenhum estudo foi publicado. Foi ainda constatado que os primeiros estudos publicados nas fontes adotadas datam do ano de 2000 (E15, E36) e o período com maior número de publicações se deu entre 2004 e 2006 (37\%).

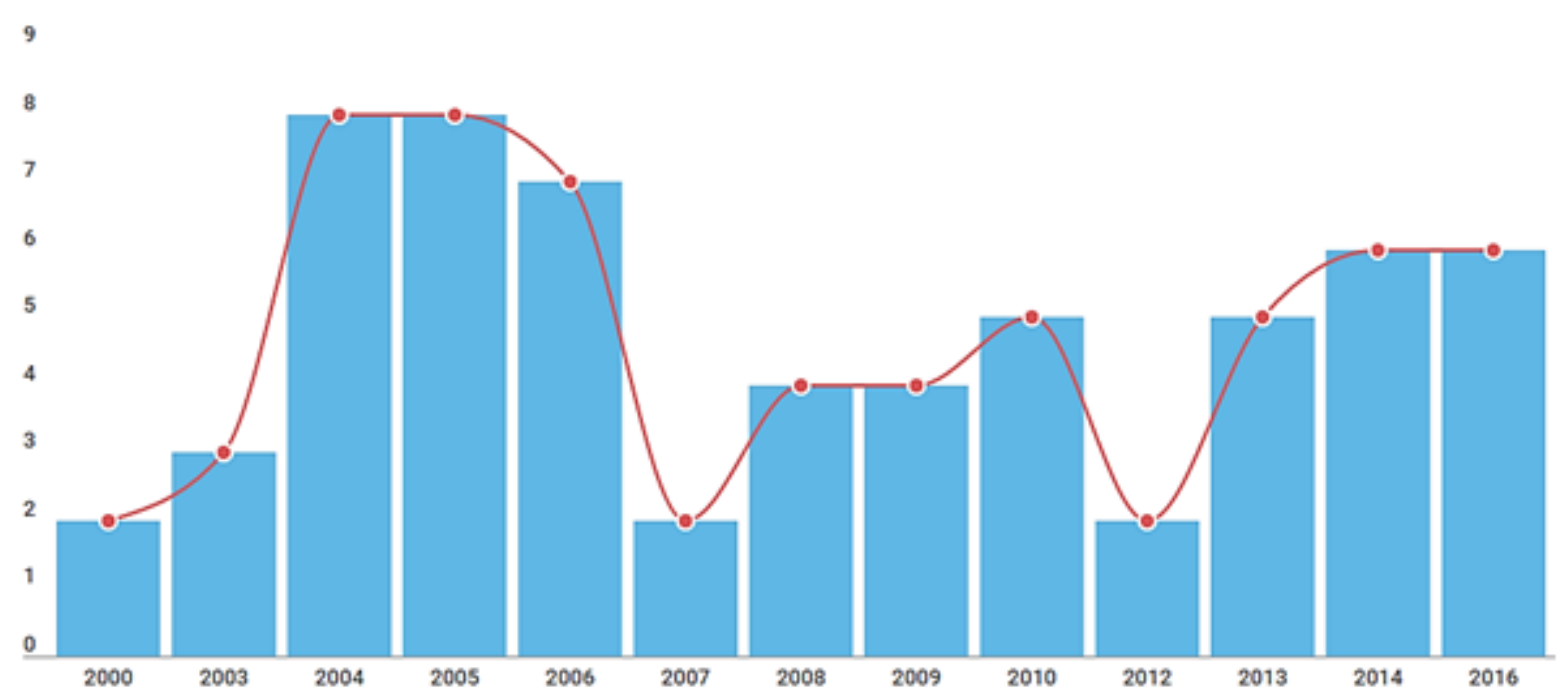

Figura 2: Estudos incluídos no MSL por ano de publicação

Com o objetivo de verificar a recorrência de termos utilizados pelos autores nas palavras-chave (keywords), foi gerado um gráfico do tipo wordcloud ou tagcloud (Figura 3). 
Nesse tipo de gráfico, a quantidade de tamanho dessa mesma palavra na vezes que uma palavra se repete no visualização.

conjunto (frequência) determinará 0

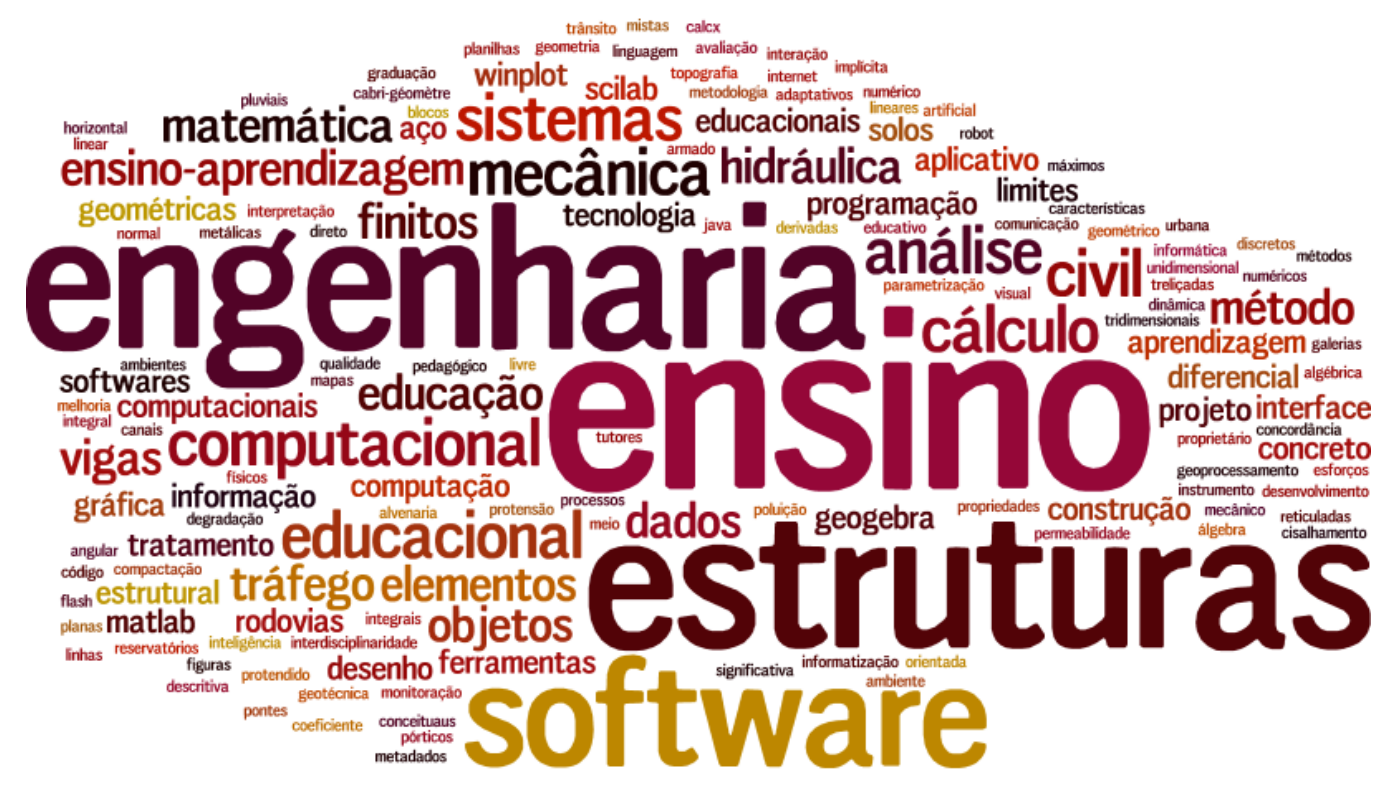

Figura 3: Palavras mais frequentes dentre as keywords (palavras-chave) dos trabalhos

Cabe ainda mencionar que 39\%

dos estudos traziam propostas de

software, ou seja, ferramentas

desenvolvidas pelos próprios autores para ensino de componentes curriculares específicos.

3.1. QP1 - Quais softwares têm sido utilizados no ensino de Engenharia Civil no Brasil?

Nos estudos investigados neste MSL foram identificados 55 softwares Engenharia Civil no Brasil. A Tabela 6 apresenta os mais frequentes. que têm sido utilizados no ensino de

Tabela 6: Softwares mais frequentes nos trabalhos analisados

\begin{tabular}{ll}
\hline NOME DA FERRAMENTA & \multicolumn{1}{c}{ ESTUDOS } \\
\hline Matlab & E18, E43, E45, E46, E49, E8 \\
Excel & E17, E23, E50 \\
Scilab & E27, E62, E8 \\
Ftool & E17, E52 \\
GDFácil & E42, E51 \\
Geogebra & E39, E40 \\
GEOWEB & E25, E47 \\
Mathematica & E53, E59 \\
Winplot & E13, E44 \\
\hline
\end{tabular}


A lista completa dos softwares catalogados encontra-se disponível online no endereço
Na Figura 4 é possível observar quantos estudos abordam cada uma das 55 ferramentas catalogadas.

https://goo.gl/KZ6M9T.

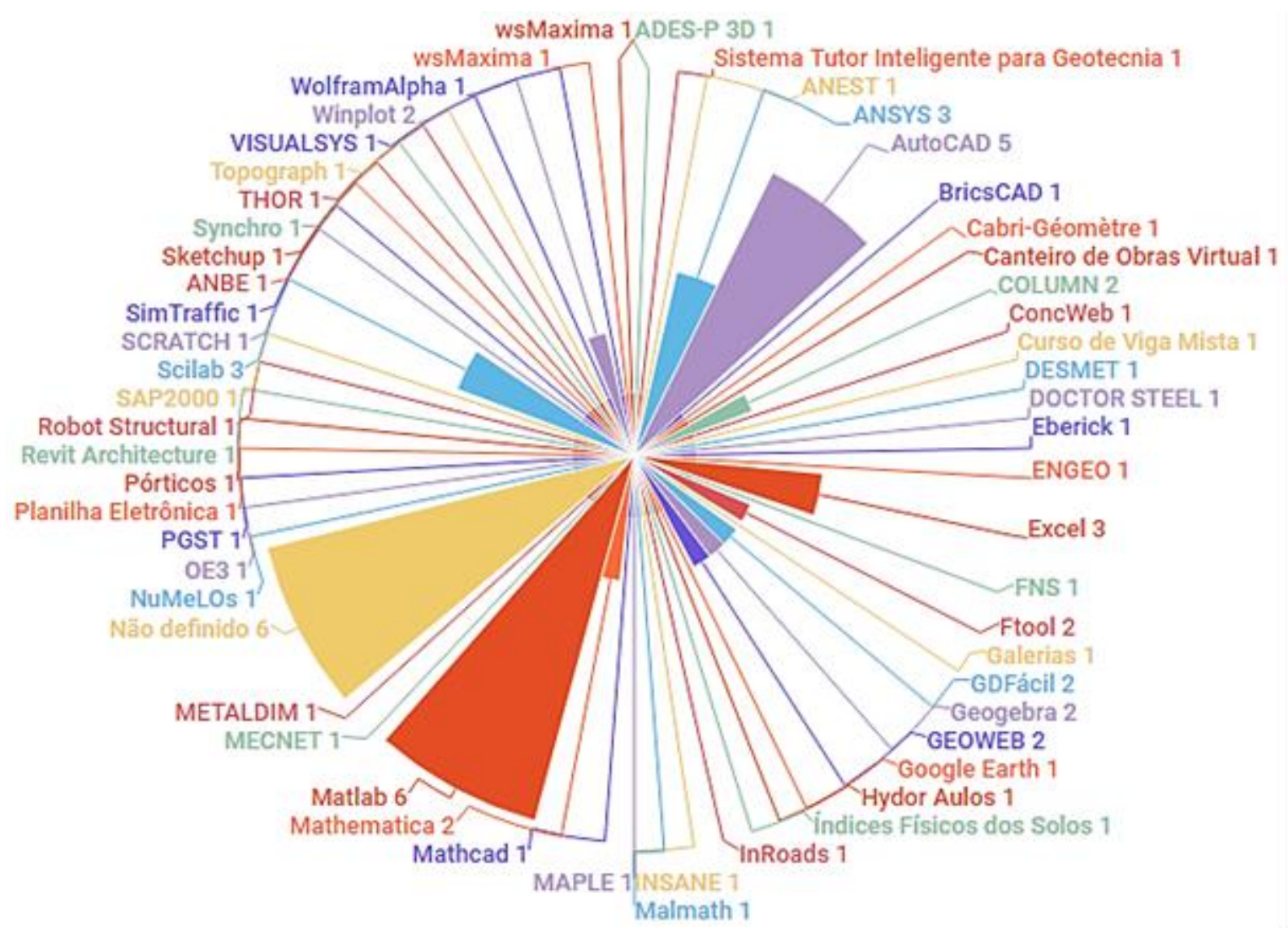

Figura 4: Softwares identificados e quantidade de estudos que os abordam

3.2QP2 - Em quais temas/disciplinas dos cursos de Engenharia Civil o uso dos softwares é mais frequente?

Analisando os dados da tabela de extração, foram constatadas que as áreas (temas ou disciplinas) da Engenharia Civil nas quais softwares identificados foram mais utilizados são: Cálculo; Estruturas; Estruturas Metálicas; Geotecnia; Mecânica dos Solos; Análise de Estruturas; Desenho
Técnico.

Ressalta-se que a denominação dos temas é baseada nos termos utilizados pelos autores em seus trabalhos, dessa maneira alguns itens podem ser subtópicos de outros na lista. 
visão completa e mais intuitiva a respeito da QP2.

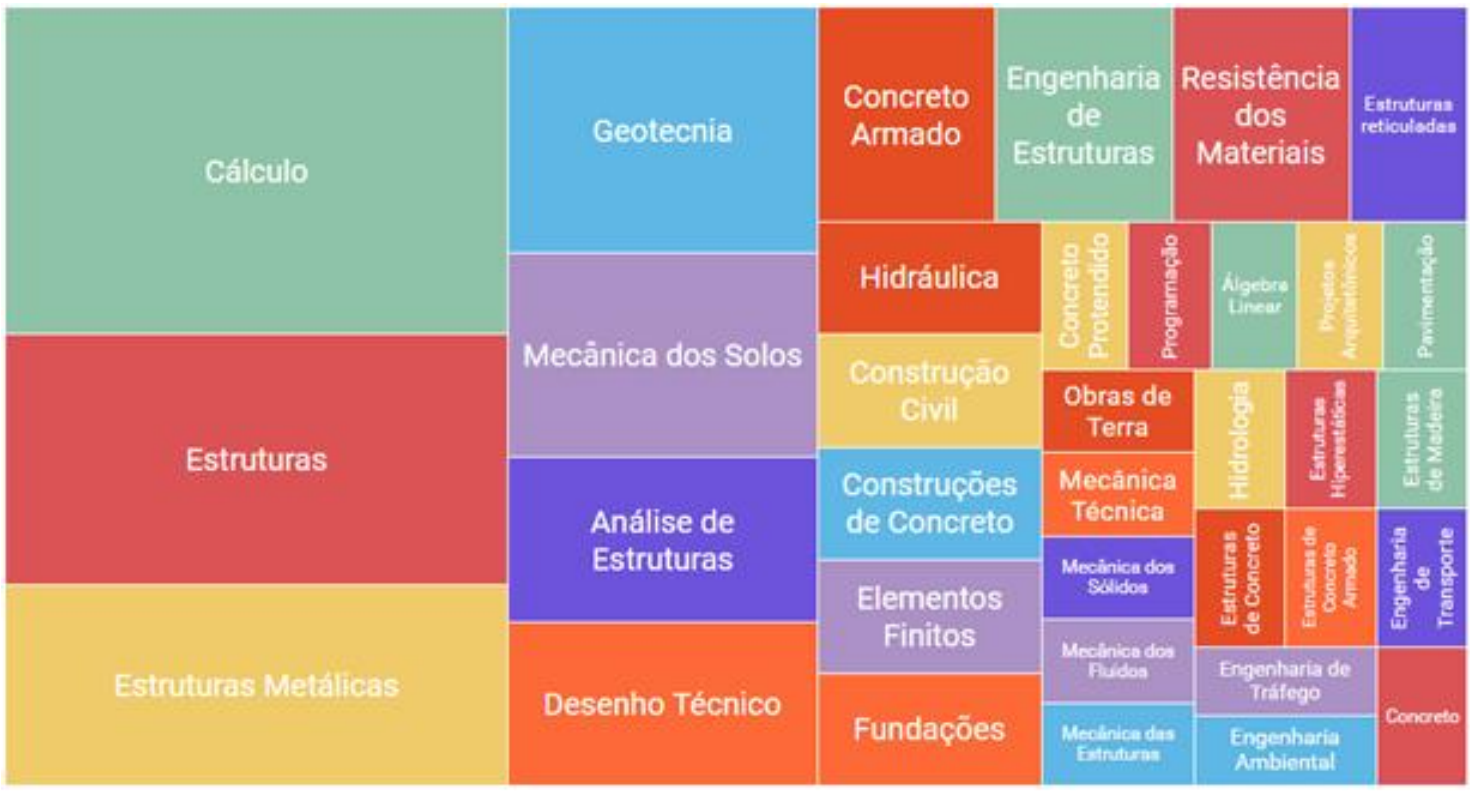

Figura 5: Disciplinas ou temas de Engenharia Civil abordados nos softwares identificados

3.3QP3 - Quais tipos de interface ou tecnologia de interação usuário-computador são usadas nos softwares identificados na QP1?

Visto que a interface é um fator de grande impacto na eficácia dos softwares (especialmente naqueles utilizados em ambientes educacionais), este MSL examinou as tecnologias de interação utilizadas nos softwares estudados.

Foi constatado que $50 \%$ dos softwares utilizaram Interface Gráfica do Usuário (GUI - Graphical User Interface) 2D, a qual faz uso de imagens e outros elementos bidimensionais em adição ao texto para representação das informações e ações disponíveis ao usuário. Em 45\% dos softwares é utilizada GUI mista (2D e 3D). Apenas em dois estudos (E3 e E4) foi observado uso exclusivo de GUI $3 \mathrm{D}$.

3.4QP4 - Quais as plataformas dos softwares identificados na QP1?

Com relação às plataformas, foi observado que houve predominância de softwares desenvolvidos para desktop (87\%), seguidos daqueles para ambiente web (E22, E25, E28, E31, 
E36, E47, E54).

Dos trabalhos incluídos na pesquisa, apenas 0 estudo E60

\section{CONSIDERAÇÕES FINAIS}

O MSL apresentado neste trabalho foi conduzido conforme a metodologia de uma Revisão Sistemática, com a intenção de ser uma revisão formal, rigorosa e confiável, mostrando-se adequado para a obtenção de estudos relevantes, gerando dados necessários para responder às questões de pesquisa.

De maneira geral, os diversos ramos da engenharia fazem uso constante das ferramentas computacionais. Com a Engenharia Civil não é diferente e, em especial, na formação dos novos engenheiros, é fundamental a utilização ampla e bem fundamentada.

Evidenciou-se que apesar de relativamente baixo, o número de publicações trazendo relatos de utilização de software no ensino de Engenharia Civil ou ainda desenvolvimento de aplicações computacionais com tal propósito se manteve constante durante o período analisado. relatava o uso ferramentas em ambiente mobile (celulares, smartphones, etc).

Um dos aspectos identificados neste MSL foi que tecnologias como realidade virtual e realidade aumentada ainda são pouco utilizadas no âmbito nacional dos softwares ou propostas de softwares para ensino do Engenharia Civil. Outra carência detectada foi o baixo de número de estudos relatando experiências com dispositivos móveis (smartphones e tablets).

Percebemos que a aplicação das ferramentas se dá em diversas disciplinas além daquelas do eixo essencial (como Física e Matemática), todavia com um uso sinérgico do conteúdo. Nesse contexto, onde os alunos são instigados para a utilização concomitante dos conhecimentos e experiências adquiridos na pluralidade de suas disciplinas, torna-se evidente a importância da abordagem interdisciplinar no processo de ensinoaprendizagem.

Por fim, cabe ressaltar que somente a utilização de softwares educacionais não cria a melhor 
situação de aprendizado. Tais ferramentas devem ser servir de forma complementar ao que se aprende em sala de aula, possibilitando uma construção do conhecimento a partir de suas experiências adquiridas (RAMIRO; COSTA e BERNARDES,
2014).

Almeja-se que os resultados apresentados nessa pesquisa forneçam uma visão abrangente acerca do tema, expondo ainda possibilidades de uso e de desenvolvimento de novas ferramentas.

\section{REFERÊNCIAS}

CASTELLS, M.: La era de la información: economía, sociedad y cultura. Fin del Milenio, Madrid, Alianza Editorial, 1998.

DOMINICINI, W. K.; COELHO, L. H. Desenvolvimento de Software Educacional para Ana'lise e Dimensionamento de Estruturas em Concreto Protendido. In: Congresso Brasileiro de Ensino de Engenharia. [s.n.], 2014. Disponível em: (http://www.abenge.org.br/cobenge-2014/Artigos/129002.pdf).

JANTSCH, A. P.; BIANCHETTI, L. Interdisciplinaridade: Para além da Filosofia do Sujeito. 3 ed. Petrópolis: Vozes, 1999.

KITCHENHAM, B. Procedures for Performing Systematic Reviews. Keele, UK, Keele University, v. 33, n. 2004, p. 1-26, 2004.

KITCHENHAM, B.; CHARTERS, S. Guidelines for Performing Systematic Literature Reviews in Software Engineering. Durham, 2007. Dispon'ivel em: (https://www.cs.auckland.ac.nz/ mria007/Sulayman/Systematic reviews 5 8.pdf).

LAGOS, P. S. Ingeniería de Software Educativo, Teorías y Metodologías que la Sustentan. Revista Ingeniería Informática, n. 6, 2000.

MODLER, L. E. A.; FEIL, M. S. Características, Possibilidades e Limitações de um Software como Ferramenta de Apoio para o Ensino das Disciplinas de Curso de Engenharia Civil da UNIJUÍ. In: Congresso Brasileiro de Ensino de Engenharia. [s.n.], 2003. Disponível em: (http://www.abenge.org.br/CobengeAnteriores/2003/artigos/ EIT615.pdf).

NICOLESCU, B. O Manifesto da Transdisciplinaridade. São Paulo: Triom, 1999.

ORMONDE, P. C. et al. Software Educacional Livre para Análise Não Linear de Rev. Cereus, v. 9, n. esp, p.02-18, ago-dez./2017, UnirG, Gurupi, TO, Brasil. 
Pórticos Planos em Estruturas Metálicas. Dissertação (Mestrado) - Universidade Federal de São Carlos, 2013. Disponível em: (https://repositorio.ufscar.br/bitstream/handle/ufscar/4687/5600.pdf).

PETERSEN, K. et al. Systematic Mapping Studies in Software Engineering. In: Proceedings of the 12th International Conference on Evaluation and Assessment in Software Engineering. Swindon, UK: BCS Learning \& Development Ltd., 2008. (EASE'08), p. $68-77$ Disponível em: (http://dl.acm.org/citation.cfm?id=2227115.2227123).

RAMIRO, F. S.; COSTA, L. A.; BERNARDES, J. A. Softwares Educacionais: Seu Uso e Importancia no Ensino-Aprendizagem dos Alunos de Engenharia Civil. [s.n.], 2014. Disponível em: http://198.136.59.239/ abengeorg/cobenge2014/Artigos/129044.pdf).

SILVA, J. G. S. et al. Uma Nova Concepção para o Ensino de Estruturas de Aço na FEN/UERJ. Revista de Ensino de Engenharia, v. 24, n. 1, 2005. Disponível em: (http://www.abenge.org.br/revista/index.php/abenge/article/view/24). 